\section{A retrospective analysis to determine the effect of independent treatment centres on the case mix for microsurgical training}

A Barsam, CJ Heatley, V Sundaram and NMG Toma

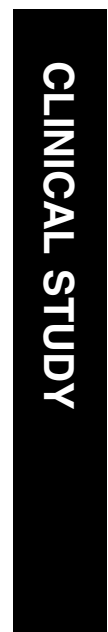

results of this study are considered in any current or future plans for ISTC continuation and expansion.

Eye (2008) 22, 687-690; doi:10.1038/sj.eye.6702718; published online 2 February 2007

Keywords: cataract surgery; phacoemulsification; case mix; training; independent treatment centres

\section{Introduction}

Cataract extraction and intraocular lens insertion is the most commonly performed surgical intervention in developed countries. The number of individuals affected by cataract and undergoing cataract surgery is expected to increase dramatically over the next 20 years as the population ages. ${ }^{1}$

In 2000 the Department of Health, as part of the action on cataracts, outlined strategies for reducing waiting times for cataract surgery. ${ }^{2}$ There have been significant changes in the provision of cataract surgery over the last few years to facilitate this goal. One such change is the utilisation of Independent Sector Treatment Centres (ISTCs). In September 2003, one such ISTC was awarded the contract to carry out 41600 cataract procedures over a 5-year period in a mixture of mobile and fixed site units throughout the UK. The Royal College of Ophthalmologists have several concerns regarding the effect of ISTCs on local National Health Service (NHS) hospitals. ${ }^{3}$ One major concern is the potential impact that ISTCs may have on the training of junior ophthalmologists.
Department of

Ophthalmology, Queen Elizabeth II Hospital, Howlands, Welwyn Garden City, UK

Correspondence: A Barsam, Department of Ophthalmology, Queen Elizabeth II Hospital, Howlands, Welwyn Garden City, Herts AL7 4HQ, UK

Tel: 01707365049 Fax: 01707391334 E-mail: abarsam@ hotmail.com

Received: 7 May 2006 Accepted in revised form: 12 December 2006 Published online: 2 February 2007 
At present ISTCs are not involved in training junior ophthalmologists. It has been suggested that ISTCs select more routine cases to provide a cost-effective service. As a consequence of this, local NHS hospitals may be left with a residual case mix of more complex patients who may be unsuitable for junior training. ${ }^{3}$

As a means to determine the impact of ISTCs on microsurgical training, a novel scoring protocol for stratification of case suitability was devised. This was used to compare all patients undergoing cataract surgery under a single consultant surgeon in a district general eye unit in a given period when a local ISTC was established with an equivalent period preceding the establishment of the ISTC. The null hypothesis that ISTCs do not affect the case mix for microsurgical training was tested.

\section{Methods}

At the beginning of August 2004, 465 patients who had been listed for cataract surgery in a district general NHS eye unit between February and July 2004 were selected to undergo their cataract surgery at a local ISTC. These patients would otherwise have been scheduled to undergo cataract surgery between the end of August and the beginning of December 2004 in the NHS eye unit. There was no other local treatment centre or waiting list initiative in operation at this time. The selection guidelines utilised by local NHS managers in combination with the ISTC was not to select any patient specifically listed for the consultant to do. As well as this, 10 further patients were deemed unsuitable by the ISTC and returned to the NHS eye unit for surgery. All patients who underwent cataract surgery on a single consultant dedicated training list in the eye unit between September and November 2004 were retrospectively analysed. These patients are representative of cases remaining after ISTC selection, that is the residual case mix. As a comparison a retrospective analysis was performed of patients who underwent cataract surgery in the NHS eye unit on the same consultant dedicated training list in the same period in 2003 when there was no ISTC or other waiting list initiative in operation. All patients were listed by the single consultant responsible for the training list.

To make a quantitative comparison between patients in the 2 years, we developed a novel scoring protocol for the stratification of cases suitable for microsurgical training.

This was based on:

1. A questionnaire sent out to all ophthalmic consultants, associate specialists, specialist registrars and senior house officers in two separate training units in a 1 year period $(n=24)$. This asked them to rank the preoperative features of an eye or patient that they thought make a case unsuitable for training.

2. A Medline review of the literature pertaining to complications of phacoemulsification cataract surgery in order to identify those characteristics that have been shown to increase the risk of intraoperative and postoperative complications. ${ }^{4-17}$

3. The Royal College of Ophthalmologists recommendation for current training requirements as well as the planned curriculum for ophthalmic specialist training (the run-through training planned as part of modernising medical careers). ${ }^{18-19}$

This protocol is outlined in Table 1 .

The sum of all categories and the corresponding point score was taken to classify patients in terms of their suitability for training. This is outlined in Table 2 .

All patients in the study were scored according to this protocol. Statistical analysis was performed using Prism 4(GraphPad Software Inc).

\section{Results}

Data were available for 129 patients, of whom 73 patients underwent cataract surgery in 2003 and 56 patients underwent cataract surgery in the same period in 2004.

Table 1 Scoring protocol for the stratification of cases suitable for microsurgical training

\begin{tabular}{ll}
\hline Category A (no points) & No unsuitability factors \\
Category B (1/2 point each) & Glaucoma, diabetic retinopathy, ARMD \\
Category C (1 point each) & $\begin{array}{l}\text { Previous vitrectomy, corneal opacification (mild), shallow AC, deep AC, age }>88 \text { years, high } \\
\text { ametropia ( }>6 \mathrm{D} \text { spherical equivalent), high astigmatism ( }>3 \mathrm{D}), \text { posterior capsular plaque. }\end{array}$ \\
& $\begin{array}{l}\text { Deep set eyes } \\
\text { Small pupil, posterior polar cataract, corneal guttata, corneal opacification (moderate,severe), } \\
\text { miscellaneous risk (eg, postural considerations), complications with previous eye, BCVA in }\end{array}$ \\
other eye $<6 / 12$ and $>6 / 24$ from irreversible aetiology. \\
Category D (2 points each) & $\begin{array}{l}\text { Dense/total } / \text { white or brunescent cataract, pseudoexfoliation, phacodonesis, history of trauma } \\
\text { to eye, BCVA in other eye }<6 / 24 \text { and }>6 / 36 \text { from irreversible aetiology. }\end{array}$ \\
Category F (5 points) & Only eye (BCVA $=6 / 36$ or less in other eye from irreversible aetiology, e.g., amblyopia)
\end{tabular}

Abbreviations: ARMD, age-related macular degeneration; BCVA, best-corrected visual acuity. 
Table 2 Classification system for suitability of cases based on score for varying levels of trainee experience

\begin{tabular}{ll}
\hline 1 Point and above & $\begin{array}{l}\text { Unsuitable for most junior trainees. Suitable for senior SHOs and above (year 2-3 and above of } \\
\text { run-through training) }\end{array}$ \\
2 Points and above & Unsuitable for SHOs. Suitable for year $1 \mathrm{SpR}$ and above (year 3-4 and above of run-through training) \\
3 Points and above & Unsuitable for junior SpRs. Suitable for year 3-4 SpR and above (years 6-7 of run-through training) \\
5 Points and above & Consultants only
\end{tabular}

Abbreviations: SHO, senior house officer; SpR, Specialist Registrar.

Table 3 The percentage of patients in each group that would be suitable for trainees at various levels of experience

\begin{tabular}{lcc}
\hline$\%$ patients unsuitable for & 2003 & 2004 \\
\hline First year SHOs & $39.7 \%(29)$ & $62.5 \%(34)$ \\
All SHOs & $19.2 \%(14)$ & $53.6 \%(29)$ \\
Junior SpRs & $9.6 \%(7)$ & $26.8 \%(15)$ \\
All except consultant & $5.5 \%(4)$ & $19.6 \%(11)$ \\
\hline
\end{tabular}

Abbreviations: SHO, senior house officer; SpR, Specialist Registrar.

There was no significant difference in baseline demographics between the two groups. Mean age was 75.3 years for 2003 compared with 75.2 years for 2004 $(P=0.93)$. The ratio of female:male for 2003 was 1.6 and for 2004 was 1.9. The ratio of first eye cataract surgery: second eye cataract surgery for 2003 was 1.5 and for 2004 was 2.1. Mean best corrected visual acuity in the eye not undergoing surgery (other eye) was 0.22 for the 2003 group compared with 0.24 for the 2004 group $(P=0.75)$.

In the 2003 group, $4.1 \%$ of cases were listed specifically for the consultant to perform and in the 2004 group $28.6 \%$ cases were listed specifically for the consultant to perform. The percentage of patients in each group that would be suitable for trainees at various levels of experience is illustrated in Table 3. Using the devised scoring protocol for the stratification of cases suitable for microsurgical training, the mean score in the 2003 group was $1.08 \pm 1.75$ (range, $0.0-10.5$ ) and for the 2004 group the mean score was $2.31 \pm 2.65$ (range, 0.0-14.5). A Mann-Whitney test showed that there was a statistically significant difference between the scores in the two groups $(P=0.0009)$.

\section{Conclusion}

In recent years there has been significant concern about the state of microsurgical training in ophthalmology.

A survey of senior house officers in the UK highlighted that of those who had completed 2 or more years' training, only $42 \%$ met the Royal College of Ophthalmologists minimum requirement of 50 complete intraocular operations performed under supervision per year. ${ }^{20}$ Similar concerns have been expressed in Australia $^{21}$ and the United States. ${ }^{22}$ Training standards must be maintained in the face of increasing patient expectations, increasing litigation, increasing complexity of surgery, and reduction of trainee hours and exposure to surgical cases.

The scoring protocol for stratification of cases suitable for microsurgical training is a novel system. Although it was devised to quantify data for statistical analysis to address our study aim, we feel it will also be an invaluable tool for both trainer and trainee alike in assessing the suitability of different cases for training. Although this is a robust and dynamic system, we acknowledge that not all trainees at a particular level of training have the same level of microsurgical aptitude. Nonetheless this system could be easily adapted to take such variation into account.

This study shows the implementation of a local ISTC resulting in a reduction of opportunity for microsurgical training. From the year preceding the introduction of the ISTC to the next year when the ISTC was recruiting patients off the waiting list, the mean unsuitability score doubled. With ISTC implementation the percentage of cases suitable only for consultants increased fourfold.

This study looked at one particular ISTC and the effect on a single hospital. Although it is likely that the effect demonstrated in this study may apply elsewhere, the results cannot necessarily be generalised to other units.

With the introduction of modernising medical careers, in order to provide seamless run-through training, the length of time the average trainee ophthalmologist has in order to learn microsurgical skills is likely to be shortened. The European Working Time Directive also ensures that in many units where trainees do 'week of nights' on call the exposure to dedicated theatre time is shortened. Presently, specialist registrars at Moorfields Eye Hospital lose 2 weeks of potential surgical training in every 4 month block owing to a week of nights and required rest time afterwards. This coupled with the decrease in suitable cases for training as shown in this study is likely to have serious consequences for microsurgical training in the UK.

We recommend that the results of this study are taken into account in any current or future plans for ISTC continuation and expansion. If ISTCs recruit patients from local NHS eye units involved in training then the trainees associated with these units should have the 
opportunity to access supervised microsurgical training within the ISTC itself.

\section{Acknowledgements}

The results of this study were presented at the 2006 Oxford Ophthalmological Congress.

\section{References}

1 Congdon N, Vingerling JR, Klein BE, West S, Friedman DS, Kempen J et al. Prevalence of cataract and pseudophakia/ aphakia among adults in the United States. Arch Ophthalmol 2004; 122(4): 487-494.

2 Action on cataracts; good practice guidance. Department of Health. www.dh.gov.uk/assetRoot/04/01/45/ 04014514.pdf.

3 Press statement on Independent Treatment Centres, November 2005 The Royal College of Ophthalmolgistswww.rcophth.ac.uk/about/press/.

4 Muhtaseb M, Kalhoro A, Ionides A. A system for preoperative stratification of cataract patients according to risk of intraoperative complications: a prospective analysis of 1441 cases. Br J Ophthalmol 2004; 88(10): 1242-1246.

5 Kuchle M, Viestenz A, Martus P, Handel A, Junemann A, Naumann GO. Anterior chamber depth and complications during cataract surgery in eyes with pseudoexfoliation syndrome. Am J Ophthalmol 2000; 129: 281-285.

6 Drolsum L, Haaskjold E, Sandvig K. Phacoemulsification in eyes with pseudoexfoliation. J Cataract Refract Surg 1998; 24: 787-792.

7 Chakrabarti A, Singh S, Krishnadas R. Phacoemulsification in eyes with white cataract. J Cataract Refract Surg 2000; 26: 1041-1047.

8 Brazitikos P, Tsinopoulos I, Papadopoulos N, Fotiadis K, Stangos NT. Ultrasonographic classification and phacoemulsification of white senile cataracts. Ophthalmology 1999; 106: 2178-2183.

9 Singh R, Vasavada A, Janaswamy G. Phacoemulsification of brunescent and black cataracts. J Cataract Refract Surg 2001; 27: $1762-1769$.
10 Lacalle V, Garate F, Alday N. Phacoemulsification cataract surgery in vitrectomized eyes. J Cataract Refract Surg 1998; 24: 806-809.

11 Pinter S, Sugar A. Phacoemulsification in eyes with past pars plana vitrectomy: Case-control study. J Cataract Refract Surg 1999; 25: 556-561.

12 Vasavada A, Singh R. Phacoemulsification in eyes with posterior polar cataract. J Cataract Refract Surg 1999; 25: 238-245.

13 Osher R, Yu BC-Y, Koch D. Posterior polar cataracts: a predisposition to intraoperative capsular rupture. J Cataract Refract Surg 1990; 16: 157-162.

14 Berler DK. Intraoperative complications during cataract surgery in the very old. Trans Am Ophthalmol Soc 2000; 98: 127-132.

15 Blair CJ, Ferguson Jr J. Exacerbation of senile macular degeneration following cataract extraction. Am J Ophthalmol. 1979; 87(1): 77-83.

16 Krepler K, Biowski R, Schrey, Jandrasits K, Wedrich A. Cataract surgery in patients with diabetic retinopathy: visual outcome, progression of diabetic retinopathy, and incidence of diabetic macular oedema. Graefes Arch Clin Exp Ophthalmol. 2002; 240(9): 735-738.

17 Yasutani H, Hayashi K, Hayashi H, Hayashi F. Intraocular pressure rise after phacoemulsification surgery in glaucoma patients. J Cataract Refract Surg. 2004; 30(6): 1219-1224.

18 The Royal College Of Ophthalmologists Training Committee Requirements. www.rcophth.ac.uk/docs/ training/logbook_information.doc.

19 The Royal College Of Ophthalmologists Draft Curriculum For Modernising Medical Careers. www.rcophthdraftcurriculum.pwp.blueyonder.co.uk.

20 Watson MP, Boulton MG, Gibson A, Murray PI, Moseley MJ, Fielder AR. The state of basic surgical training in the UK: ophthalmology as a case example. J R Soc Med 2004; 97(4): 174-178.

21 Ormonde SE. Ophthalmic microsurgical training: are we approaching a crisis point? Clin Experiment Ophthalmol 2005; 33(5): 453-454.

22 Rowden A, Krishna R. Resident cataract surgical training in United States residency programs. J Cataract Refract Surg 2002; 28: 2202-2205. 